\title{
RELATIONS BETWEEN THE GRAMIAN, THE WRONSKIAN, AND A THIRD DETERMI- NANT CONNECTED WITH THE PROBLEM OF LINEAR DEPENDENCE.
}

BY PROFESSOR D. R. CURTISS.

(Read before the American Mathematical Society, April 28, 1911.)

The three determinants here discussed, the Gramian $G$, the Wronskian $W$, and the third determinant $D$, are formed for $n$ functions, real or complex, $f_{1}, f_{2}, \cdots, f_{n}$, which we shall here suppose for simplicity to be functions of a real variable $x$, as follows :

$$
G=\left|\begin{array}{cccc}
\int_{a}^{b} f_{1}(x) \bar{f}_{1}(x) d x & \int_{a}^{b} f_{1}(x) \bar{f}_{2}(x) d x & \ldots & \int_{a}^{b} f_{1}(x) \bar{f}_{n}(x) d x \\
\int_{a}^{b} f_{2}(x) \bar{f}_{1}(x) d x & \int_{a}^{b} f_{2}(x) \bar{f}_{2}(x) d x & \ldots & \int_{a}^{b} f_{2}(x) \bar{f}_{n}(x) d x \\
\vdots & \vdots & \ldots & \vdots \\
\int_{a}^{b} f_{n}(x) \bar{f}_{1}(x) d x & \int_{a}^{b} f_{n}(x) \bar{f}_{2}(x) d x & \cdots & \int_{a}^{b} f_{n}(x) \bar{f}_{n}(x) d x
\end{array}\right|
$$

where $\bar{f}(x)$ is the conjugate of $f(x)$;

$$
\begin{gathered}
W=\left|\begin{array}{cccc}
f_{1}(x) & f_{2}(x) & \cdots & f_{n}(x) \\
\frac{d}{d x} f_{1}(x) & \frac{d}{d x} f_{2}(x) & \cdots & \frac{d}{d x} f_{n}(x) \\
\vdots & \vdots & \cdots & \vdots \\
\frac{d^{n-1}}{d x^{n-1}} f_{1}(x) & \frac{d^{n-1}}{d x^{n-1}} f_{2}(x) & \cdots & \frac{d^{n-1}}{d x^{n-1}} f_{n}(x)
\end{array}\right| \\
D=\left|\begin{array}{cccc}
f_{1}\left(x_{1}\right) & f_{2}\left(x_{1}\right) & \cdots & f_{n}\left(x_{1}\right) \\
f_{1}\left(x_{2}\right) & f_{2}\left(x_{2}\right) & \cdots & f_{n}\left(x_{2}\right) \\
\vdots & \vdots & \cdots & \vdots \\
f_{1}\left(x_{n}\right) & f_{2}\left(x_{n}\right) & \cdots & f_{n}\left(x_{n}\right)
\end{array}\right|
\end{gathered}
$$


It is well known * that if the functions $f_{1}, f_{2}, \cdots, f_{n}$ are continuous (or even more general conditions may be given), the vanishing of $G$ is a necessary and sufficient condition for their linear dependence in the interval $[a, b]$. The identical vanishing of $W$ is a necessary condition provided the derivatives involved exist, and with certain added hypotheses it is also sufficient. $\dagger$

It has perhaps been previously noted, though I have been able to find no explicit reference on this point, $\$$ that the identical vanishing of $D$ for all values of the independent variables $x_{1}, x_{2}, \cdots, x_{n}$ in $[a, b]$ is a necessary and sufficient condition that $f_{1}(x), f_{2}(x), \cdots, f_{n}(x)$ be linearly dependent in $[a, b]$, i. e., that there exist a linear relation valid at every point of $[a, b]$

$$
c_{1} f_{1}(x)+c_{2} f_{2}(x)+\cdots+c_{n} f_{n}(x)=0,
$$

where at least one of the constants $c$ is not zero. That $D \equiv 0$ is a necessary condition is obvious; to see this, if the notation is so chosen that $c_{n} \neq 0$, we have only to multiply the $i$ th column of $D$ by $c_{i} / c_{n}(i=1,2, \cdots, n-1)$ and add to the last column, thus making each element of that column zero on account of (1). Conversely, if $D \equiv 0$, suppose the minor of the element $f_{n}\left(x_{n}\right)$, which we may denote by $D_{n}$, is not zero for at least one set of values of $x_{1}, x_{2}, \ldots, x_{n-1}$ in $[a, b]$. Such a value being given to these variables we have a relation (1) if we replace $x_{n}$ by $x$ and expand $D$ in terms of the elements of the last row and their minors. If $D_{n} \equiv 0$ in $[a, b]$, then either the same is true of $f_{1}(x)$, in which case (1) will hold if $c_{1} \neq 0, c_{2}=\cdots$ $=c_{n}=0$, or else there is a determinant formed by suppressing the last $m$ rows and the last $m$ columns of $D$ which vanishes identically in $[a, b]$ but in which the minor of the last element of the last row is different from zero for at least one set of values of the variables concerned in $[a, b]$. This determinant is of the same type as $D$, and arguing as before we establish a linear relation between $f_{1}, f_{2}, \cdots, f_{m}$, which may be regarded as a relation (1) where $c_{m+1}=\cdots=c_{n}=0$.

* Cf. G. Kowalewski, Einführung in die Determinantentheorie, Chap. XV.; also Bôcher's review of this chapter in the BuLLETIN, vol. 17 (1910), p. 126.

†Cf. in addition to the above citations a paper by the author, "The vanishing of the Wronskian and the problem of linear dependence," Mathematische Annalen, vol. 65 (1908), p. 282, where further references are given.

$\ddagger$ Possibly this is implied in a remark of the paper of Richardson and Hurwitz referred to on page 464 . 
If we compare the criteria for linear dependence furnished by the vanishing of these three determinants, we see that the condition $D \equiv 0$ applies in all cases where the functions are defined for every point of $[a, b]$, while $G=0$ requires the continuity, or at least the integrability, of the functions concerned, and $W \equiv 0$ not only implies the existence of derivatives of order up to $n-1$ but is even then insufficient without additional hypotheses. We may note also that the criteria $D \equiv 0$ and $G=0$ apply for functions of more than one variable, with suitable change of notation, while no single identity of the same sort can replace $W \equiv 0$.

A certain interest attaches to formulas connecting $G, D$, and $W$. Thus Meder has recently proved the relation*

$$
\left.\frac{d^{m^{2}} G}{d b^{m^{2}}}\right|_{b=a}=M[W(\alpha)]^{2},
$$

where $M$ is a constant, and $W(\alpha)$ indicates the Wronskian formed for $x=a$. This formula cannot, however, be reversed so as to express $G$ in terms of $W$. We now proceed to obtain relations connecting $D$ and $G$, and $D$ and $W$, by combining two of which $G$ may be expressed in terms of $W$.

An identity connecting $D$ and $G$ may be derived at once, in case the functions $f_{1}, f_{2}, \cdots, f_{n}$ are all real, from the formula (4) given by Richardson and Hurwitz in a recent article in the Bulletin. + If we put $k=1$, this formula becomes, with appropriate changes in notation,

$$
G=\frac{1}{n !} \int_{a}^{b} \cdots \int_{a}^{b} D^{2} d x_{1} \cdots d x_{n} .
$$

It can easily be shown that if the functions concerned are complex the corresponding formula is

$$
G=\frac{1}{n !} \int_{a}^{b} \ldots \int_{a}^{b} D \bar{D} d x_{1} \ldots d x_{n}
$$

where $\bar{D}$ is the conjugate of $D$. These formulas put in evidence the property $G \geqq 0$, and give a simple proof that $G=0$

* " Ueber den Zusammenhang zwischen den Determinanten von Gram und Wronski," Monatshefte für Mathematik und Physik, vol. 21 (1910), p. 336. + "Note on determinants whose terms are certain integrals," vol. 16 (1909), no 1 , p. 14 . 
is a necessary and sufficient condition for linear dependence. They are valid, with suitable changes of notation, for functions of any number of variables.

To express $W$ in terms of $D$, formulas (2) and (3) or $\left(3^{\prime}\right)$ may be combined; or we may verify directly the result

$$
W(x)=\left[\frac{\partial^{\frac{1}{n}(n-1)}}{\partial x_{2} \partial x_{3}^{2} \cdots \partial x_{n}^{n-1}} D\right]_{x_{1}=x_{2}=\ldots=x_{n}=x} .
$$

The problem of finding a formula which will give $D$ in terms of $W$ in such a way as to put in evidence the vanishing of $D$ when $W \equiv 0$ in $[a, b]$ is impossible of solution except under added hypotheses, since $W \equiv 0$ is not alone a sufficient condition for linear dependence. If, however, one of the $n-1$ rowed determinants of the matrix formed by the first $n-1$ rows of $W$ vanishes at no point of $[a, b]$, for example the determinant $W_{n}$ formed by suppressing the last column of this matrix, we may obtain a relation of the kind desired with the aid of the well-known formula of Frobenius for Wronskians.* If we use the notation $W_{r}(x)$ to indicate the determinant formed by suppressing the last row and the $r$ th column of $W$, and $W_{r, s}(x)$ for the determinant formed by suppressing the last row and the sth column of $W_{r}$, a special case of Frobenius' formula may be written

$$
\begin{aligned}
& W(x) \cdot W_{n, r}(x)=W_{n}(x) \frac{d}{d x} W_{r}(x)-W_{r}(x) \frac{d}{d x} W_{n}(x) \\
&(r=1,2, \cdots, n-1) .
\end{aligned}
$$

Since $W_{n}(x) \neq 0$ in $[a, b]$, we may divide $(5)$ by $\left[W_{n}(x)\right]^{2}$ and integrate, thus obtaining

$$
\begin{aligned}
& \int_{z}^{x} \frac{W(\xi) W_{n, r}(\xi)}{\left[W_{n}(\xi)\right]^{2}} d \xi=\frac{W_{r}(x)}{W_{n}(x)}-\frac{W_{r}(z)}{W_{n}(z)} \\
&(r=1,2, \cdots, n-1) .
\end{aligned}
$$

If we replace the last row of $W$ by the first, so that the resulting determinant vanishes identically, we have, on expanding according to the minors of this new last row,

$$
\sum_{r=1}^{n}(-1)^{r-1} f_{r}(x) W_{r}(x)=0
$$

\footnotetext{
${ }^{*}$ Cf. Crelle, vol. 77 (1874), p. 248 ; also footnote, p. 284, of the article of the author, cited on p. 463.
} 
or, since $W_{n}(x) \neq 0$ in $[a, b]$,

$$
f_{n}(x)=\sum_{r=1}^{n-1}(-1)^{r+n-1} f_{r}(x) \frac{W_{r}(x)}{W_{n}(x)} .
$$

The substitution of (6) in this last relation gives

$$
\begin{aligned}
& f_{n}(x)=\int_{z}^{x} \frac{W(\xi)\left[\sum_{r=1}^{n-1}(-1)^{r+n-1} f_{r}(x) W_{n, r}(\xi)\right]}{\left[W_{n}(\xi)\right]^{2}} d \xi \\
& +\frac{\sum_{r=1}^{n-1}(-1)^{r+n-1} f_{r}(x) W_{r}(z)}{W_{n}(z)} .
\end{aligned}
$$

It may be noted that the expression in brackets in the numerator of the integrand in (7) is equal to the determinant

$$
\Delta_{n}(x, \xi)=\left|\begin{array}{cccc}
f_{1}(x) & f_{2}(x) & \cdots & f_{n-1}(x) \\
-f_{1}(\xi) & -f_{2}(\xi) & \cdots & -f_{n-1}(\xi) \\
\vdots & \vdots & \cdots & \vdots \\
-\frac{d^{n-2} f_{1}(\xi)}{d \xi^{n-2}} & -\frac{d^{n-2} f_{2}(\xi)}{d \xi^{n-2}} & \cdots & -\frac{d^{n-2} f_{n-1}}{d \xi^{n-2}}
\end{array}\right|
$$

To obtain the desired result we have now only to replace the elements $f_{n}\left(x_{1}\right), f_{n}\left(x_{2}\right), \cdots, f_{n}\left(x_{n}\right)$ of the last column of $D$ by the corresponding expressions $(7)$, multiply the elements of the $r$ th column by $(-1)^{r+n-1} \cdot W_{r}(z) / W_{n}(z)$ for each value of $r$ from 1 to $n-1$, and subtract from the last column. This gives us

(8) $D=\left|\begin{array}{ccccc}f_{1}\left(x_{2}\right) & f_{2}\left(x_{2}\right) & \cdots & f_{n-1}\left(x_{2}\right) & \int_{z}^{x_{2} W(\xi) \Delta_{n}\left(x_{2}, \xi\right)} \frac{}{\left[W_{n}(\xi)\right]^{2}} d \xi \\ \vdots & \vdots & \cdots & \vdots & \vdots \\ f_{1}\left(x_{n}\right) & f_{2}\left(x_{n}\right) & \cdots & f_{n-1}\left(x_{n}\right) & \int_{z}^{x_{n}} \frac{W(\xi) \Delta_{n}\left(x_{n}, \xi\right)}{\left[W_{n}(\xi)\right]^{2}} d \xi\end{array}\right|$

where $z$ is any point of $[a, b]$.

Formula (8) may be transformed in a number of ways which we shall not discuss here. It should be remarked that 
if the integral is taken in the ordinary or Riemannian sense, we must presuppose the integrability of the expressions $W(x) \cdot W_{n, r}(x) /\left[W_{n}(x)\right]^{2}$, but this is not necessary if the Lebesgue integral is used, provided, at least, the above expression is finite in $[a, b]$. The condition $W_{n}(x) \neq 0$ in $[a, b]$ may also be removed in certain cases, as when $W_{n}(x)$ vanishes at only a finite number of points, $x_{0}, x_{1}, \ldots, x_{m}$ in $[a, b]$ so that $\lim _{x=x_{i}} W_{r}(x) / W_{n}(x)$ exists and is finite for $\left(\begin{array}{c}r=0,1, \ldots, n-1 \\ i=0,1, \ldots, m\end{array}\right)$. It seems unlikely, however, that formula (8) can be so extended as to give new criteria for linear dependence.

In conclusion we note that formulas (3), or $\left(3^{\prime}\right)$, and (8) taken together express $G$ in terms of $W$ in such a way as to show that $G=0$ when $W$ vanishes, under the restrictions named. We thus have what may be regarded as the converse of (2). Similarly (8) and (2) express $D$ in terms of $G$.

\section{NoRTHWESTERN UNIVERSITY, March 3, 1911.}

\section{NOTE ON INTEGRATION OF SERIES BY LEBESGUE INTEGRALS.}

BY MR. W. A. WILSON,

LebesGue has shown in his work on integration that if a limited function, $f(x) \geqq 0$, is measurable for a measurable field $A$, it is "summable," or possesses a Lebesgue integral, and the value of this integral is the measure of the ordinate set $Y$, whose points are defined by the conditions : $x$ in $A, 0 \leqq y \leqq f(x)$. The converse is also true; that is, if $Y$ is measurable, $f(x)$ is measurable and

$$
\text { meas } Y=\int_{A} f(x) d A \text {. }
$$

A proof of this may be found in Schoenfliess, Jahresbericht der Deutschen Mathematiker-Vereinigung, Ergänzungsband II, part II, page 320 .

It is the purpose of this note to show how by use of this idea the proof of Lebesgue's theorem on termwise integration of series can be greatly simplified and reduced to elementary theorems on point aggregates. The theorem in question is proved in Hobson's Theory of Functions of a Real Variable, 